\title{
Éditorial
}

\section{Hommage au Dr Daniel Viennet}

\author{
Chers collègues,
}

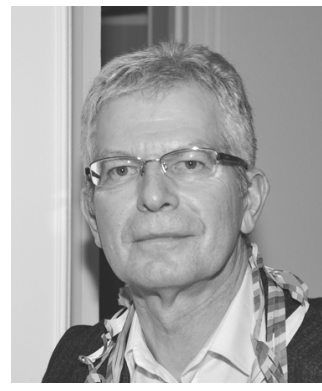

Le département de chirurgie buccale de la faculté d'odontologie de Nancy a le regret de vous annoncer le décès du docteur Daniel Viennet survenu le jeudi 2 juillet, à la suite d'une longue maladie.

Né en 1952 à Besançon, il a découvert la chirurgie orale durant ses études à la faculté d'odontologie de Nancy sous l'impulsion du Pr Durivaux. Dès lors, son intérêt pour cette discipline n'a jamais cessé. Il exerçait son métier avec beaucoup de minutie, de rigueur et de passion, s'avérant avide de connaissances et de savoir. Il a travaillé en secteur libéral mais aussi hospitalier aux côtés du Dr Wang, dans le service de chirurgie maxillo-faciale du CHU de Nancy. Ses patients appréciaient non seulement ses qualités professionnelles mais aussi son écoute et son empathie.

Aimant la transmission du savoir et grand pédagogue, Daniel était maître de conférence des universités et praticien hospitalier des centres de soins et de recherche dentaire depuis 1988 à la faculté d'odontologie de l'université de Lorraine. Ses collègues ainsi que les nombreux étudiants qu'il a formés ont pu apprécier son professionnalisme, sa disponibilité ainsi que sa gentillesse. Très aimé par les étudiants, qu'il accompagnait durant leurs premiers pas de clinicien, il est à l'origine de nombreuses thèses d'exercices.

Il était investi tant dans la vie universitaire locale que nationale, aussi bien au sein du CNU de chirurgie buccale, pathologie et thérapeutique, anesthésiologie et réanimation que de la Société francophone de chirurgie orale. Fort de cette expérience, il a préparé et soutenu de nombreux jeunes praticiens souhaitant embrasser la carrière hospitalo-universitaire.

Il avait choisi d'occulter la maladie, d'avoir « une vie normale » et de poursuivre son activité professionnelle, et ce, toujours avec le sourire. Son départ laisse un grand vide au sein de la faculté d'odontologie et plus particulièrement au sein du département de chirurgie orale. Nous espérons être à la hauteur des valeurs qu'il nous a transmises tout au long de notre cursus, tant sur le plan clinique que pédagogique.

Tous ceux qui l'ont côtoyé vous diront qu'il était d'un naturel optimiste. Épicurien, fin gourmet, il aimait la nature et plus particulièrement la chasse, il collectionnait les lettres anciennes et les marques postales, et il savait apprécier les petites choses de la vie. Diane et Sarah, ses deux filles, ainsi que ses deux petits enfants, Gaspard et Daphné, étaient sa plus grande fierté. Il n'aura malheureusement pas pu bénéficier de sa retraite qu'il comptait passer auprès de sa famille, en jouant son rôle de « papoune ».

Toutes nos pensées vont à ses proches, à ses filles ainsi qu'à son épouse Maryse qui l'a accompagné durant ce combat contre la maladie.
Le département de chirurgie orale de la faculté d'odontologie de Nancy 